\title{
Epidural anesthesia for management of pseudo aneurysm of femoral artery in ASA- IV patient on dual antiplatelet therapy: Case report and a paradigm shift in anaesthesia practice
}

\author{
Abeer A. Sherif, MD(1), Abdulatif Araji, MD(2) \\ (1) Professor of anaesthesia \& Pain medicine, Faculty of Medicine, El-menufia university, Consultant \& Chief anaesthesiologist NMC \\ royal hospital UAE \\ (2) Vascular surgeon, NMC Royal hospital, UAE
}

\begin{abstract}
Introduction: Anticoagulation achieved with dual antiplatelet therapy is becoming increasingly common after coronary angioplasty and represents an everyday challenge to the anaesthesiologists when neuraxial anaesthesia is to be considered specially in high risk patients with multiple comorbidities. Epidural hematoma is a rare but potentially devastating complication of neuraxial anesthesia. To date, no clear data addressing the safety of epidural anesthesia in patients on dual antiplatelet therapy. Despite being extremely rare, the development of epidural hematoma is a known risk after epidural analgesia. The third National Audit Project of the Royal College of Anesthetists found an incidence of two in 100000 cases. The incidence of spontaneous epidural hematoma is rarer, estimated at 1 per 1000000 patients per year, most going undetected. Antiplatelet agents are known to be protective in most patients at increased risk of occlusive vascular events, including those with an acute myocardial infarction, ischemic stroke, angina, peripheral arterial disease, or atrial fibrillation. Brilianta (ticagrelor) is a platelet adenosine diphosphate (ADP) P2Y12 receptor inhibitor, a new oral anticoagulants are approved for a variety of clinical syndromes like coronary artery disease, peripheral vascular disease, or ischaemic strokes. Current ASRA recommendations to stop Brilianta (Ticagrelor) therapy 5 - 7 days before the surgery are based on level III evidence such as clinical judgement and sporadic case reports of epidural hematoma after regional analgesia, in patients with a history of taking Tecagrelor in combination with other anticoagulants ( level $C$ evidence). To date, no prospective studies have investigated the safety of epidural analgesia in patients actively taking Ticagrelor.
\end{abstract}

Methods: A 80-year-old man presented with a history of swelling and redness in his left groin. Patients was on aspirin and brilianta after angioplasty 3 month ago, ischemic nephropathy, COPD, sever chest infection, heart failure and pacemaker in place. Epidural anesthesia was planned because of the poor general condition of the patient. Epidural was conducted in the sitting position using $L$ 3- 4 interspaces, $10 \mathrm{ml}$ of bupivacaine $0.5 \%$ was injected in the epidural catheter. Primary repair of the femoral vein performed, along with the patching of the left common femoral artery. The surgical procedure lasted for 6 hours and the patient was shifted to ICU for monitoring. The intraoperative as well as the postoperative period remained uneventful.

\section{Discussion:}

The risk of central neuraxial blockade in patients treated with newer antiplatelet drugs is unclear. However, a disturbing lack of data exists concerning the safety of neuroaxial blockade in such patients, with a wide difference in opinion not only among anesthetists but also between published guidelines. The American Society of Regional Anesthesia agreed on an interval of $5-7$ days between discontinuation of Brilianta (Ticagrelor) therapy and neuraxial blockade. In the current case the patient had recently undergone a coronary artery stent implantation and was treated with both Brilianta, and aspirin. Despite this dual antiplatelet therapy, uneventful epidural anesthesia at two levels was carried out. Our case report demonstrate that, for certain high-risk patients, the benefit of placing an epidural catheter while actively taking antiplatelet therapy may be considered.

\section{Conclusion:}

Epidural anesthesia was conducted safely for emergency vascular surgery, in high risk patient with multiple comorbidities. Decision to perform regional anesthesia in the patients receiving antithrombotic drugs should be made on an individual basis weighing risk of epidural hematoma with the benefits of regional anesthesia. This report is not meant to recommend the liberal placement of epidural catheters in patients actively taking dual antiplatelet therapy. prospective studies with a large number of patients are needed in order to give more accurate recommendation regarding performing epidural block in such population

Table (1) Coexisting conditions in the patient

Peripheral vascular disease

Hypertension

Diabetes mellitus

Coronary artery disease

Congestive heart failure

History of cerebrovascular accidents

Peripheral neuropathy
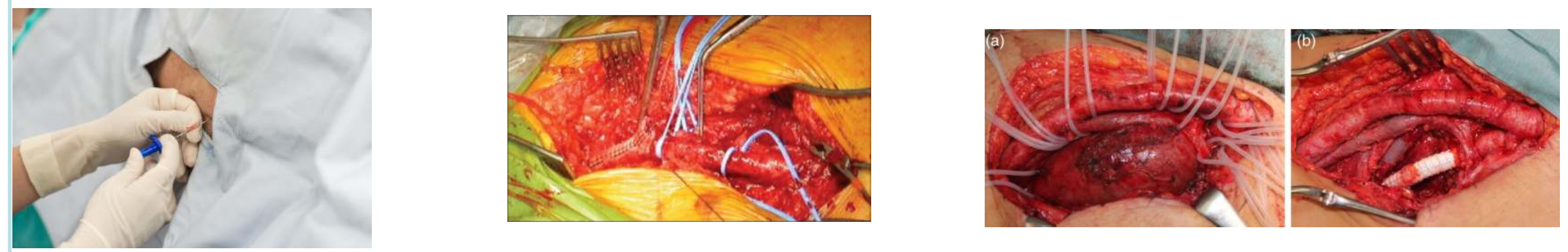